\title{
Accidental diagnosis of COVID-19 pneumonia after 18F FDG PET/CT: a case series
}

\author{
Laura Olivari ${ }^{1}\left[\right.$. Niccolò Riccardi ${ }^{2} \cdot$ Paola Rodari $^{2} \cdot$ Dora Buonfrate $^{2} \cdot$ Stefania Diodato $^{1} \cdot$ Fabio Formenti ${ }^{2}$. \\ Andrea Angheben ${ }^{2} \cdot$ Matteo Salgarello ${ }^{1}$
}

Received: 8 July 2020 / Accepted: 11 September 2020 / Published online: 24 September 2020

(c) Italian Association of Nuclear Medicine and Molecular Imaging 2020

\begin{abstract}
Purpose The aim of this case series is to illustrate possible [18F]-FDG uptake patterns associated to COVID-19.

Methods Retrospective assessment of all Fluorine-18 fluorodeoxyglucose positron emission tomography/computed tomography (18F-FDG PET/CT) scans performed for any clinical / oncological reason from 1st April 2020 to 30th April 2020. Results of PCR testing for SARS-CoV-2 were retrieved for all patients with lung consolidations and/or peripheral ground glass opacities characterized by increased metabolism to evaluate any possible association with the viral infection.

Results Seven (4\%) out of 172 FDG-PET scans were included. Six out of seven patients (85\%) had positive RT-PCR for SARS-CoV-2, while one patient (15\%) had possible (not PCR confirmed) COVID-19 pneumonia.

Conclusion Suspicious accidental COVID-19 findings in Nuclear Medicine Department need to be reported and appropriately evaluated to implement proper supportive treatment and infection control measures.
\end{abstract}

Keywords COVID-19 $\cdot$ SARS-CoV-2 $\cdot$ FDG PET/CT $\cdot$ Imaging $\cdot$ Diagnosis $\cdot$ Ground-glass opacity

\section{Introduction}

The novel beta-coronavirus, Severe Acute Respiratory Syndrome (SARS) Coronavirus (CoV)-2, the etiological agent of Coronavirus disease 2019 (COVID-19) pneumonia, is posing a threat to human beings worldwide $[1,2]$. The virus, first discovered in Wuhan (Hubei province, China) as the responsible of pneumonia of unknown origin, spreads quickly to all continents, causing more than 5.5 million cases [3]. COVID-19 took a huge toll on Italy, with more than 230,000 cases and 34,000 deaths [4] up to 1 st June 2020. Even if challenging, early identification of the severe acute respiratory illness (SARI) held by SARS-CoV-2 is crucial for patient clinical management and infection control. SARS-CoV-2 nucleic acid testing on nasal and pharyngeal

Laura Olivari

laura.olivari@sacrocuore.it

1 Department of Nuclear Medicine, IRCCS Sacro Cuore Don Calabria Hospital, Via Don Angelo Sempreboni, 5, Cap 37024 Negrar Di Valpolicella, VR, Italy

2 Department of Infectious, Tropical Diseases and Microbiology, IRCCS Sacro Cuore Don Calabria Hospital, Negrar Di Valpolicella, Verona, Italy swabs is currently the gold standard for COVID-19 diagnosis $[5,6]$, however, false-negative results can occur and, if the clinical-epidemiological suspect remains high despite a first negative swab, further evaluation for SARS-CoV-2 infection is still recommended [7]. Even asymptomatic patients may show abnormalities in chest computer tomography (CT) or chest X-Ray, performed for other clinical reasons, leading to the suspect of COVID-19 infection $[8,9]$.

Fluorine-18 fluorodeoxyglucose positron emission tomography / computed tomography (18F-FDG PET/CT) provides functional and morphological information on infectious and inflammatory diseases, and [18F]-FDG uptake is mainly related to the glycolytic activity of the cells involved in the inflammatory response [10]. Some recent case series suggest a potential role of 18F-FDG PET/CT, in evaluation of possible COVID-19 patients, as some findings may raise the index of suspicion for COVID-19-related pneumonia [11-15], regardless of the symptoms. The aim of this work is to describe 18F-FDG PET/CT patterns possibly associated with SARS-CoV-2 infection in patients undergoing the procedure for other clinical reasons. 


\section{Methods}

A retrospective study was held at IRCCS Sacro Cuore Don Calabria Hospital in Negrar (VR, Italy). For the study purpose, we reviewed all 18F-FDG PET/CT scans carried out from 1st to 30th April 2020. According to the local protocols, both outpatients and inpatients were tested for SARSCov-2 infection in case of abnormal pulmonary findings, irrespective of symptoms. Moreover, all patients underwent temperature check upon arrival at the hospital. 18F-FDG PET/CT was acquired in fasting patients, who had previous low-carbohydrate, high-fat, high-protein meal, from the skull base to the tights approximately $1 \mathrm{~h}$ after [18F]-FDG injection (activity range 200-300 MBq, according to weight) with a Siemens Biograph mCT-S (64) system (Siemens, Knoxville, TN). Images were analyzed with the Siemens SyngoTrueD 3D VOI isocontour tool (Siemens). SUVmax was obtained for each lung lesion.

Detection of nucleic acid from SARS-CoV-2 in nasopharyngeal swabs was carried out in accordance to the protocol published by Corman et al. [6], with minor modifications. For study purpose, we classified as "confirmed COVID-19 cases" those with positive PCR, while "possible COVID-19 cases" were those with abnormal radiological findings and an unidentified etiological cause.

\section{Results}

One-hundred-and-seventy-two patients underwent 18F-FDG PET/CT. Seven patients (4\%) had abnormal lung lesions. Eventually, there were six confirmed COVID-19 cases (85\%) and one possible COVID-19 case (15\%) (Table 1, Fig. 1).

\section{Case 1}

A 58-year-old female with pancreatic neuroendocrine tumor, who had pancreatoduodenectomy (G2; pT2pN1cM0) in January 2018, presented to our Nuclear Medicine Department to evaluate clinical recurrence with 68-Gallium somatostatin receptor-targeted ligand positron emission tomography/computed tomography (68 GaDOTATOC PET/CT) and 18F-FDG PET/CT.

Both examinations revealed local recurrence in the paraaortic region, while [18F]-FDG showed a single liver metastasis. Moreover, 18F-FDG PET/CT examination presented bilateral sub-segmental areas of ground glass opacities (GGO) in the lungs with increased [18F]-FDG uptake, while no pleural and mediastinal lymph nodes involvement was observed (Fig. 2a-c). These lung lesions showed no uptake in 68 Ga-DOTATOC PET/CT (Fig. 2d-f).

\section{Case 2}

An 85-year-old male was admitted to our hospital because of persistent weakness and anemia. He had cystectomy with uretero-cutaneostomy in November 2019 for bladder urothelial cancer (pT4pN0). 18F-FDG PET/CT documented cancer recurrence in the right obturator lymph node and in a common right iliac lymph node $(1.5 \mathrm{~cm}$; SUVmax 9.4). 18F-FDG PET/CT revealed lung opacities with mild uptake in subpleurical right basal region and in the middle lobe (Fig. 3a-c).

Table 1 Patient characteristics, 18F FDG PET/CT indication and Nasopharyngeal swabs results

\begin{tabular}{|c|c|c|c|c|c|c|c|}
\hline Patient's & Age & Sex & Symptoms & & FDG PET/CT indication & NP swabs & Follow up after hydroxychloroquine and \\
\hline 1 & 58 & $\mathrm{~F}$ & None & $\mathrm{R}$ & NET & Positive & Outpatient, alive \\
\hline 2 & 85 & M & None & $\mathrm{R}$ & Bladder uroterial cancer & Positive & Inpatient, discharged, alive \\
\hline 3 & 59 & $\mathrm{~F}$ & None & $\mathrm{O}$ & CLABSI & Positive & Inpatient, discharged, alive \\
\hline 4 & 62 & M & None & $\mathrm{S}$ & Renal carcinoma & Positive & Outpatient, alive \\
\hline 5 & 61 & M & None & $S$ & Lung cancer & Positive & $\begin{array}{l}\text { Inpatient, discharged, died for oncological } \\
\text { disease progression within } 3 \text { months }\end{array}$ \\
\hline 6 & 57 & $\mathrm{~F}$ & $\begin{array}{l}\text { Previous negative } \\
\text { NP swab; Mild } \\
\text { cough }\end{array}$ & $\mathrm{R}$ & DLBCL & $\begin{array}{l}\text { Negative; } \\
\text { clinician } \\
\text { treated as } \\
\text { COVID-19 }\end{array}$ & Outpatient, alive \\
\hline 7 & 71 & M & None & $\mathrm{R}$ & Tonsil squamous cell carcinoma & Positive & Outpatient, alive \\
\hline
\end{tabular}

$C L A B S I$ central line-associated bloodstream infection, $D L B C L$ diffuse large B cell lymphoma, $F$ female, $M$ male, $N P$ swabs Nasopharyngeal swabs, NET neuroendocrine tumour, $O$ non oncological disease, $S$ tumour staging, $R$ tumour re-staging in patients with clinical suspect of recurrence 
Fig. 1 Schematic representation of patients' evaluation in our Nuclear Medicine Department (a) Oncological 18F-FDG PET/ $\mathrm{CT}$ for tumour staging and tumour re-staging in patients with clinical suspect of recurrence; (b) Non-oncological 18FFDG PET/CT, included endocarditis and spondylodiscitis; (c) The RNA was extracted from clinical samples (nasopharyngeal swab using Eswab regular, Copan, Brescia-Italy) with the MagNA Pure LC2 system (Roche, Penzberg, Germany), with the MagNAPure LC RNA Isolation Kit-High Performance; $\mathrm{SpO} 2$ peripheral oxygen saturation, $R R$ respiratory rate
NUCLEAR MEDICINE DEPARTMENT TRIAGE

Check-up: SpO2, RR, temperature control with termoscan;

Symptoms screening: cough, dyspnea, anosmia, dysgeusia, diarrhea, fever $>37.5^{\circ}$

Patient History: previous contact with COVID-19 infected people.

\section{NO clinical suspect of COVID-19}

172 patients undergoing FDG-PET for

Oncological evaluation $(94 \%)^{a}$ and NON oncological evaluation (6\%) ${ }^{b}$

Lung CT scan evaluation before discharging

7 patients (4\%) Abnormal pulmonary PET-CT findings

Potentially infected patients

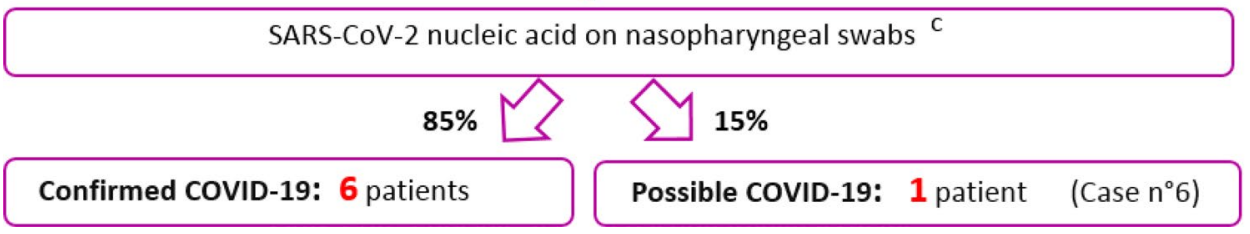

\section{Case 3}

A 59-year-old female, admitted to our hospital because of ulcerative colitis flare, underwent colectomy with ileostomy. Hospitalization was complicated by a Staphylococcus epidermidis central line-associated bloodstream infection (CLABSI). 18F-FDG PET/CT was performed to investigate a possible endocarditis. While 18F-FDG PET/CT did not show any endocarditis sign, we found hypermetabolic lungs opacities at the lingula and the right pulmonary base and hypermetabolic mediastinal lymph nodes (Fig. 3d-f).

\section{Case 4}

A 62-year-old male, with recent partial nephrectomy for clear cell papillary renal carcinoma ( $\mathrm{pT} 2 \mathrm{cN} 0)$, presented to our department for evaluation of a pre-surgical lung lesion identified with CT. 18F-FDG PET/CT revealed multiple and diffuse GGO that were not present 10 days before, at the CT (Fig. 4 A-C). CT, performed one month later of COVID-19 treatment, revealed the resolution of GGO and the persistence of the nodular lung lesion $(1.5 \mathrm{~cm}$; SUVmax 4 of the suspect lung lesion).

\section{Case 5}

A 61-year-old male presented to our Emergency Department with left hemiparesis. Contrast-enhanced CT scan revealed a right frontal lesion and a lung lesion. This patient had no fever nor cough, and 18F-FDG PET/CT was performed for lung cancer staging. 18F-FDG PET/CT revealed a $5 \mathrm{~cm}$ lung lesion with mediastinal lymph nodal involvement, right adrenal metastasis, multiple bone lesions, and confirmed the brain lesion. Moreover, 18F-FDG PET/CT showed crazy paving pattern in the lung with increased [18F]-FDG uptake (Fig. 4d-f). The patient underwent lung biopsy revealing lung adenocarcinoma.

\section{Case 6}

A 57-year-old female underwent 18F-FDG PET/CT re-staging after chemo-immunotherapy for diffuse large B cell lymphoma (DLBCL stage I, unilateral cervical nodes). 18F-FDG PET/ CT demonstrated complete metabolic oncological response, but revealed multifocal peripheral GGO with increased [18F]FDG uptake (SUV max 13) in the pulmonary right lower lobe (Fig. 5a-c). She had been tested for SARS-CoV-2 thirty days before, based on her husband positivity, and she was negative. On the day of examination, she had no fever, oxygen saturation was $>94 \%$, but she was under azithromycin for mild dry cough. After the 18F-FDG PET/CT, she repeated the SARSCov-2 test that was still negative. However, in the next two weeks, her cough worsened and she developed fever $\left(38.5^{\circ} \mathrm{C}\right)$. Therefore, hydroxychloroquine and heparin were administered, and she had rapid clinical improvement.

\section{Case 7}

A 71-year-old male underwent 18F-FDG PET/CT during the follow-up of oropharynx cancer treated with combined chemo-radiotherapy (cT2cN1). 18F-FDG PET/CT was 


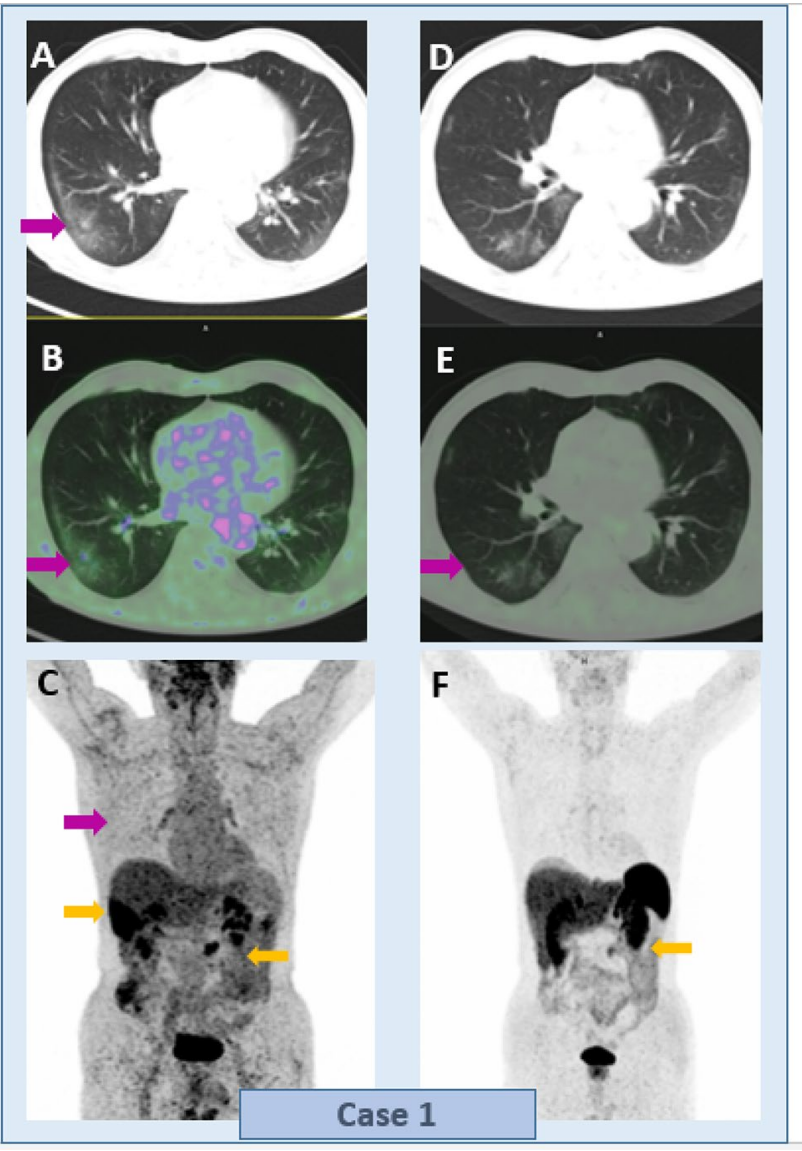

Fig. 2 a-c 18F-FDG PET/CT, performed for pancreatic neuroendocrine tumour, showed bilateral sub-segmental areas of ground glass opacities (purple arrows; SUVmax range 3.7-4). The patient had local recurrence in the paraaortic region and liver metastasis (orange arrow). Panel a: transaxial co-registered CT; Panel b: transaxial and sagittal fused PET/CT; Panel c: 18F-FDG Maximum Intensity projection (MIP). d-f 68 Ga-DOTATOC PET/CT show no uptake in lung lesion (purple arrow). The patient had local recurrence in the paraaortic region (orange arrow). Panel d: transaxial co-registered CT; Panel e: transaxial and sagittal fused PET/CT; Panel f: 68 Ga-DOTATOC Maximum Intensity projection (MIP)

negative for tumor relapse but revealed multilobar peripheral GGO in both lungs with increased metabolism (SUV max 6), strongly suggesting for COVID-19 pneumonia (Fig. 5d-f). He had mild leukopenia with lymphopenia and high level of C-reactive protein, but no history of exposure to infected people, nor COVID-19 compatible symptoms. Oxygen saturation was normal.

\section{Discussion}

We reported a case series of seven patients with 18F-FDG PET/CT showing SARS-Cov-2 associated pneumonia. COVID-19 pandemic had a major impact on health care providers, who had to deal with challenging decisions, and had to update standard operating procedures to keep delivering essential health care services. Since COVID-19 spread in Veneto Region (Italy), we have provided surgical masks to all patients attending our nuclear medicine service, and we have routinely assessed their respiratory rate, oxygen saturation and body temperature with termoscan. We interviewed every patient about COVID-19 suggestive symptoms, such as cough, dyspnea, anosmia, dysgeusia, diarrhea, fever $\left(>37.5^{\circ} \mathrm{C}\right)$ and previous contact with infected people.

Of note, in our series, we observed lung involvement caused by SARS-CoV-2 infection even in patients without symptoms. Indeed, previous studies demonstrated that CT scan is more sensible in revealing SARS-CoV-2 lung involvement before clinical symptoms appearance. Earlystage COVID-19 pneumonia presents with segmental GGO, plaques or nodules in the peripheral and posterior lungs, while disease progression leads to spread of the lesions with consolidations, crazy paving pattern, interlobular septal thickening and air bronchi sign $[8,9]$.

In our patients, $\mathrm{CT}$ findings were associated with abnormal [18F]-FDG uptake in the lungs, in accordance with few previous case series [11-15]. Lung viral infections cause diffuse alveolar damage related to host response reaction activating inflammatory cells, such as neutrophils, monocytes, $\mathrm{T}$ cell lymphocyte and macrophage. Alveoli fill with blood, water and inflammatory cells [16]. Since inflammatory cells show high expression of glucose transporter receptors (GLUT) and high levels of exochinasis, [18F]-FDG is commonly used for infectious and inflammatory diseases. However, [18F]-FDG cannot differentiate between distinct cell populations, nor is specific for viral infections [10]. Macrophage activation syndrome seems to be an important feature of COVID-19 pneumonia [18], and in the future, other tracers, such as choline, may have a potential role as hypothesized by recent papers [19-21]. To date, we are aware that the metabolic information does not provide additional data to $\mathrm{CT}$, which exhibits a more accurate pattern. However, during the outbreak of COVID-19, all abnormal lung findings detected by $18 \mathrm{~F}-\mathrm{FDG}$ PET/CT should be regarded as suspected pulmonary SARS-CoV-2 infections [22], and should prompt appropriate specific laboratory testing.

Therefore, as suggested by the recent guidance for nuclear medicine departments [23, 24], a correct and prompt assessment of chest 18F-FDG PET/CT images is necessary before discharging patients to identify potentially infected patients and put in place appropriate infection control measures. 

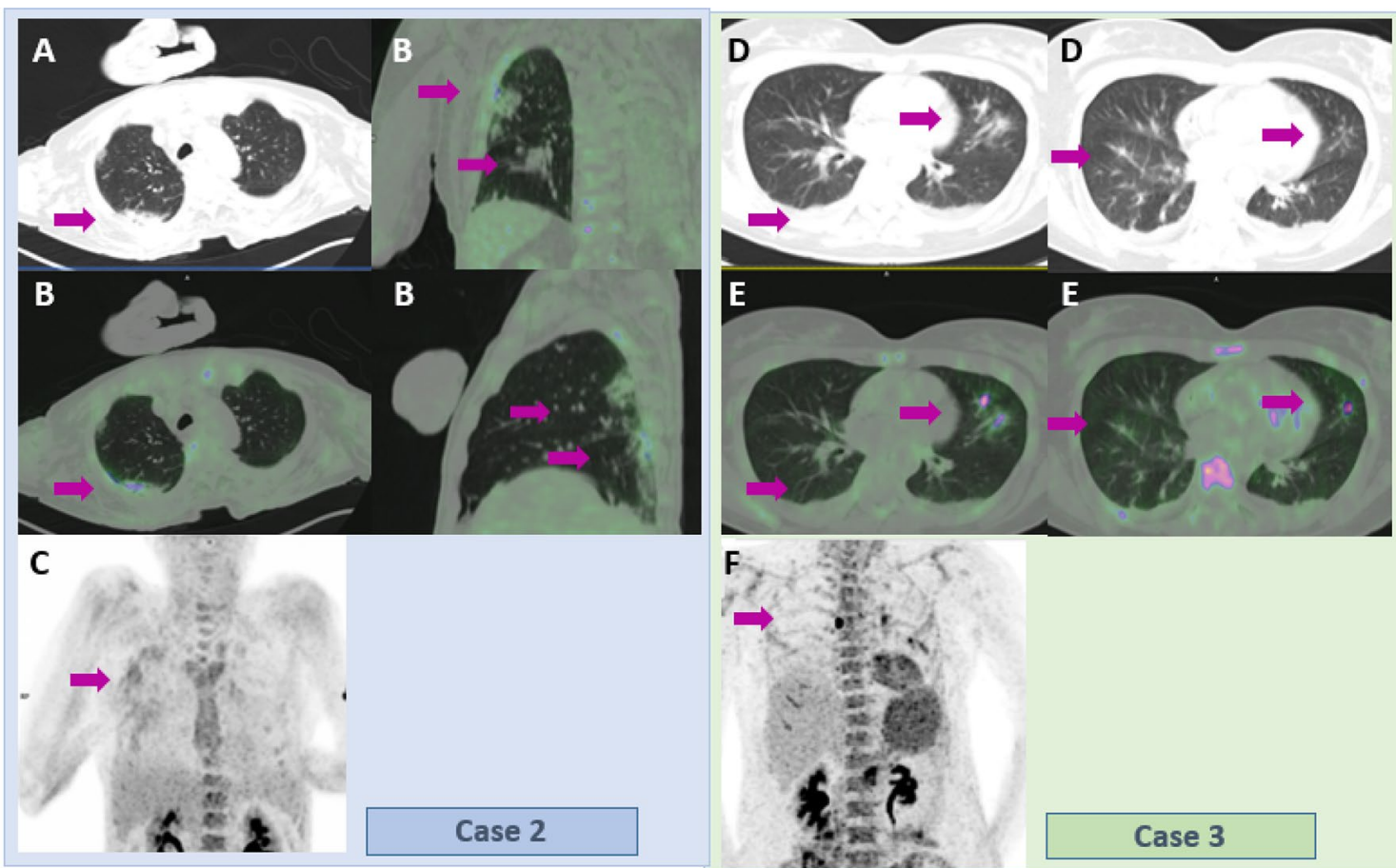

Case 2

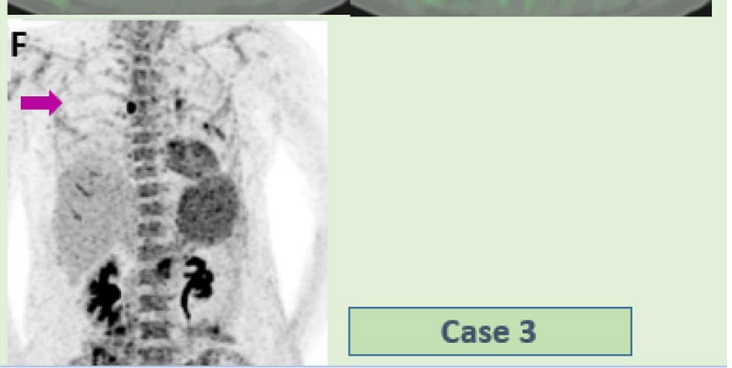

Fig. 3 a-c 18F-FDG PET/CT, performed for vesical urothelial cancer, showed lung opacities with mild uptake in subpleural right basal region and in the middle lobe were present (purple arrows; SUVmax range 3.6-4). Panel a: transaxial CT; Panel b: transaxial, coronal and sagittal fused PET/CT; Panel c: Maximum Intensity projection (MIP). d-f 18F-FDG PET/CT, performed for sepsi-endocarditis, performed after a 12-h fast and a pre-scan, showed mild interstitial consolidation-thickening (purple arrows; SUVmax range 3.8-4.3). Panel d: transaxial co-registered CT; Panel e: transaxial and sagittal fused $\mathrm{PET} / \mathrm{CT}$; Panel f: Maximum Intensity projection (MIP) 


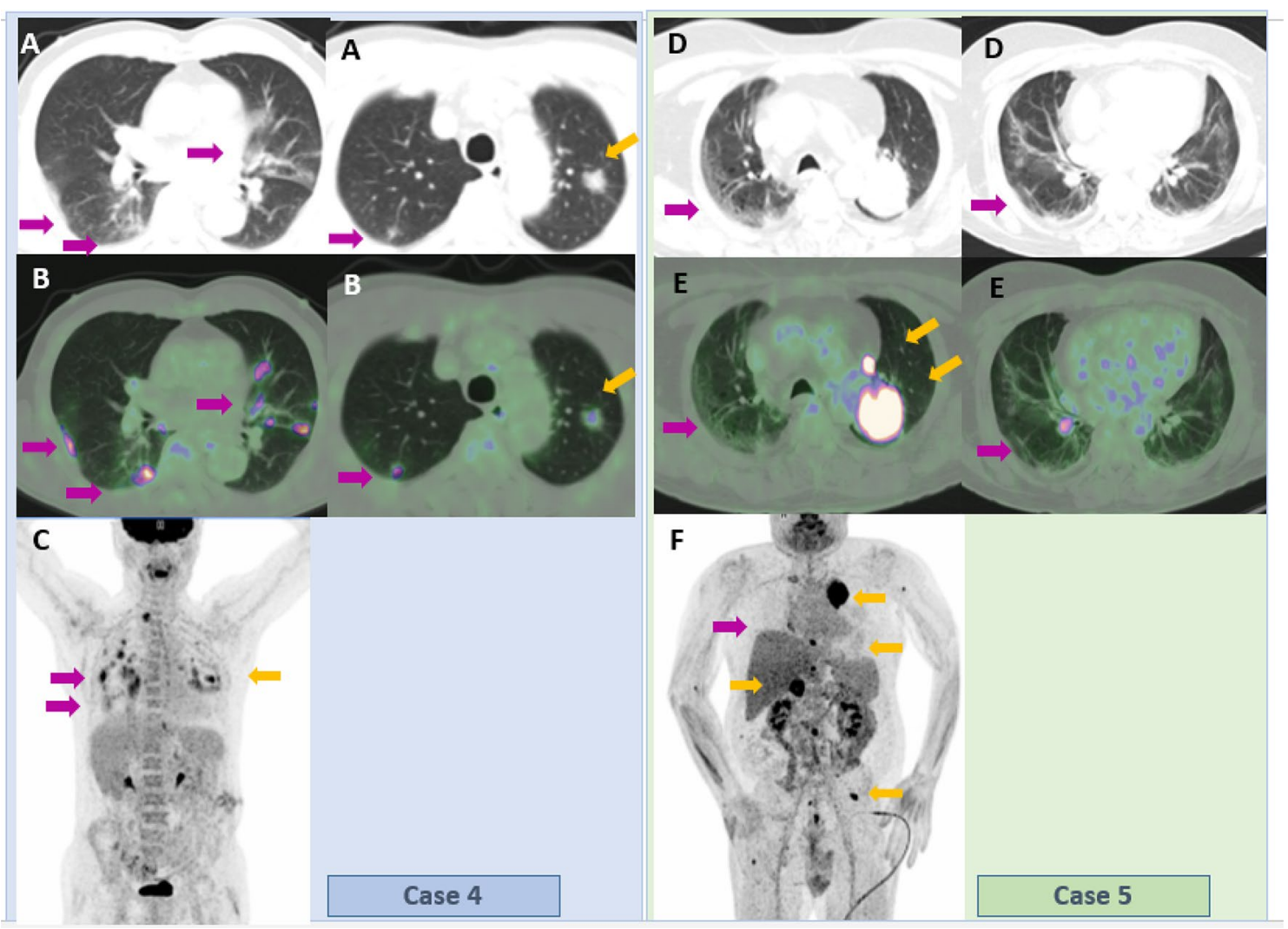

Fig. 4 a-c 18F-FDG PET/CT, performed for incidental lung lesion identified in a pre-surgical CT, showed an increased uptake in the speculated lung lesion (orange arrow; SUVmax 4.5) and multiple and diffuse GGO with increased metabolism (purple arrows; SUVmax range 5.8-7). Panel a: transaxial co-registered CT; Panel b: transaxial fused PET/CT; Panel c: Maximum Intensity projection (MIP). d-f 18 F-FDG PET/CT, performed to assess suspected lung cancer spread, showed a $5 \mathrm{~cm}$ lung lesion with mediastinal lymph nodal involvement, right adrenal metastasis, multiple bone lesions (orange arrows) and crazy paving pattern in the lung with increased FDG uptake (purple arrows; SUVmax range 4.6-5). Panel d: transaxial co-registered CT; Panel e: transaxial and sagittal fused PET/CT; Panel f: Maximum Intensity projection (MIP) 


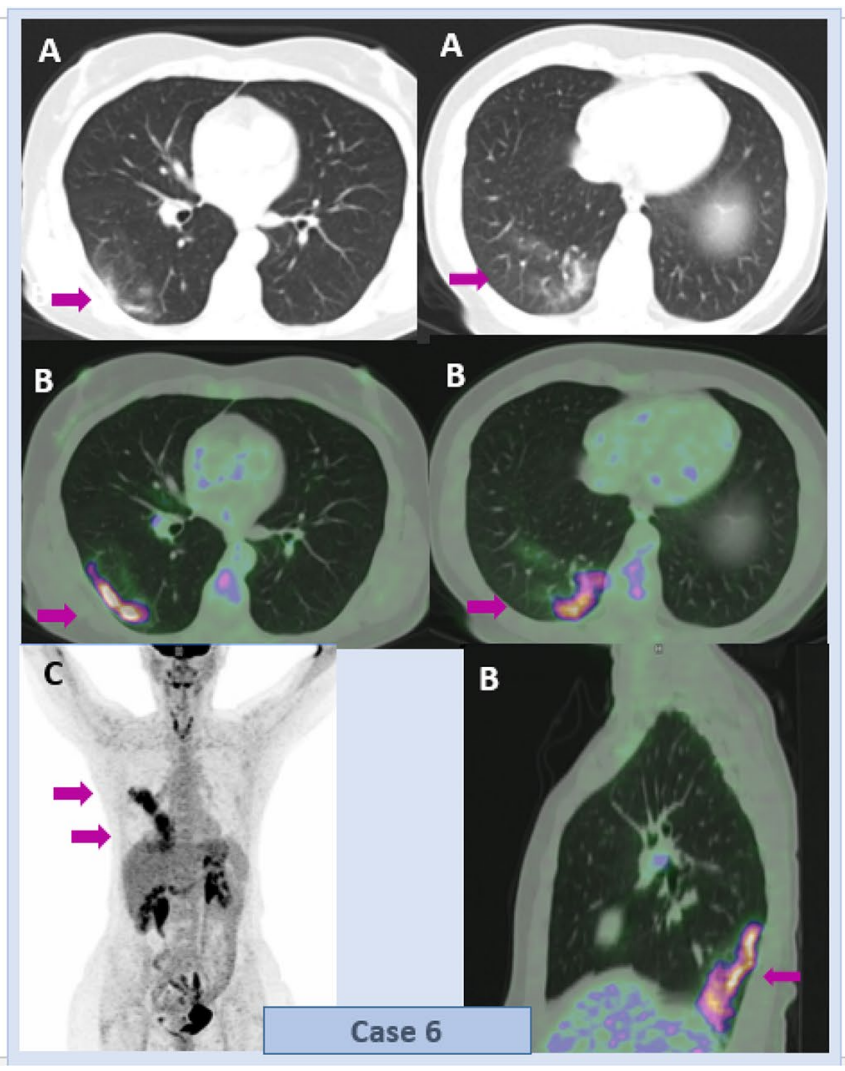

Fig. 5 a-c 18F-FDG PET/CT, performed for re-staging of DLBCL, showed multifocal peripheral GGO with increased FDG uptake (purple arrows; SUV max 13). Panel a: transaxial co-registered CT; Panel b: transaxial and sagittal fused PET/CT; Panel c: Maximum Intensity projection (MIP). d-f $18 \mathrm{~F}-\mathrm{FDG}$ PET/CT, performed for follow-up

\section{Acknowledgements Dottor Paolo Artioli and Giorgio Fagioli.}

Author contributions LO and NR contributing to conception and design; SD and LO acquired and interpreted data, LO drafted the manuscript; NR, PR, DB, AA, FF and MS revising the manuscript. All the authors approved the manuscript.

Funding This work was partly supported by the Italian Ministry of Health "Fondi Ricerca Corrente-L1P5" to IRCCS Sacro Cuore Don Calabria Hospital.

\section{Compliance with ethical standards}

\section{Conflict of interest None.}

Ethical approval This article does not contain any studies with animals performed by any of the authors. All procedures performed in studies involving human participants were in accordance with the ethical standards of the institutional and/or national research committee and with the principles of the 1964 Declaration of Helsinki and its later amendments or comparable ethical standards.

Informed consent Informed consent to assess PET-FDG was obtained from all the patients included in the study. Not all the patients could be

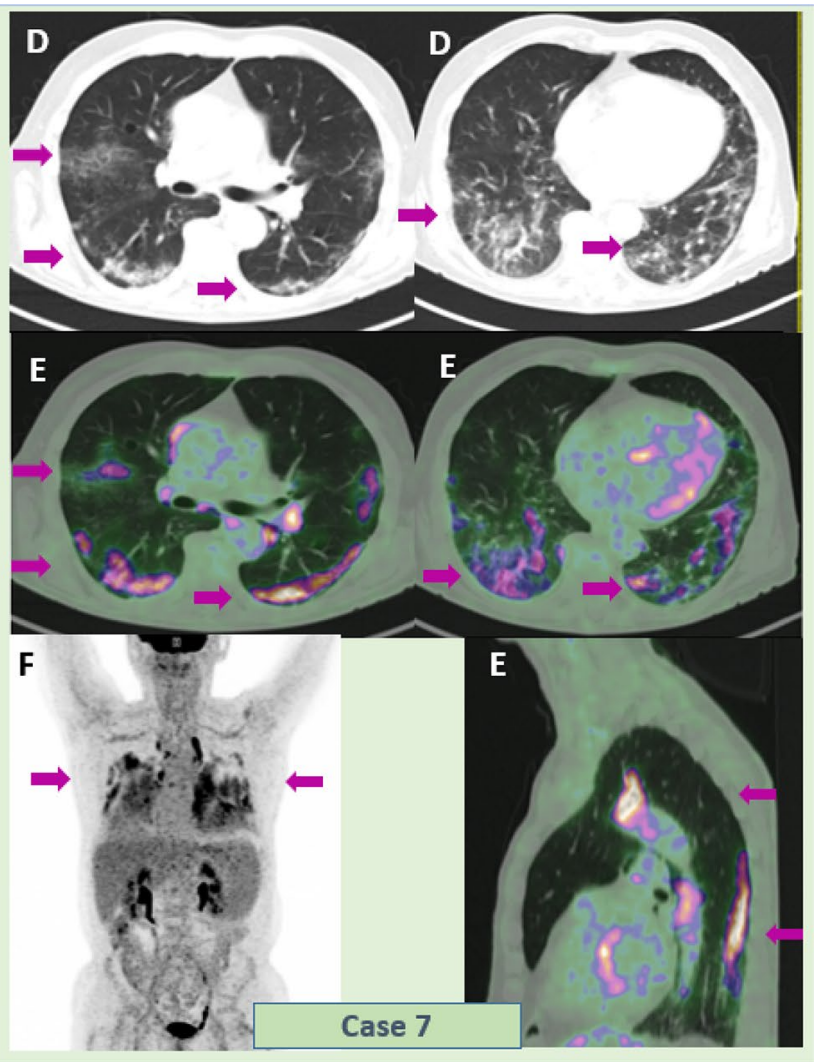

of squamous cell carcinoma of the tonsil, showed multilobar peripheral GGO in both lungs with increased metabolism (purple arrows; SUVmax range 5.3-6). Panel d: transaxial co-registered CT; Panel e: transaxial and sagittal fused PET/CT; Panel f: Maximum Intensity projection (MIP)

reach and therefore our Ethics Committee acknowledged and waived the need for consent.

Data sharing No datasets were generated or analysed during the current study.

\section{References}

1. Heymann DL, Shindo N (2020) WHO scientific and technical advisory group for infectious hazards. COVID-19: what is next for public health? Lancet 395(10224):542-545. https://doi. org/10.1016/S0140-6736(20)30374-3

2. Wang C, Horby PW, Hayden FG (2020) Gao GF (2020) A novel coronavirus outbreak of global health concern [published correction appears in Lancet. Lancet 395(10223):470-473. https://doi. org/10.1016/S0140-6736(20)30185-93

3. Johns Hopkins University. 2020. COVID-19 Dashboard by the Center for Systems Science and Engineering (CSSE) at Johns Hopkins University (JHU) https://coronavirus.jhu.edu/map.html. Accessed 1st June 2020

4. Italian Government Health Center https://www.salute.gov.it/porta le/nuovocoronavirus. Accessed 1st June 2020 
5. Guan WJ, Ni ZY, Hu Y et al (2020) Clinical characteristics of coronavirus disease 2019 in China. N Engl J Med 382(18):17081720. https://doi.org/10.1056/NEJMoa2002032(Acces sed25.05.20202020)

6. Corman VM, Landt O, Kaiser M et al (2020) Detection of 2019 novel coronavirus (2019-nCoV) by real-time RT-PCR. Euro Surveill 25(3):2000045. https://doi.org/10.2807/1560-7917. ES.2020.25.3.2000045

7. Woloshin S, Patel N, Kesselheim AS (2020) False negative tests for SARS-CoV-2 infection-challenges and implications. N Engl J Med 383(6):e38. https://doi.org/10.1056/NEJMp2015897

8. Han R, Huang L, Jiang H, Dong J, Peng H, Zhang D. Early clinical and CT manifestations of coronavirus disease 2019 (COVID-19) Pneumonia [published online ahead of print, 2020 Mar 17]. AJR Am J Roentgenol. 2020;1-6. doi:10.2214/AJR.20.22961

9. Jajodia A, Ebner L, Heidinger B, K CA, Prosch H (2020) Imaging in corona virus disease 2019 (COVID-19)-A scoping review [published online ahead of print, 2020 May 11]. Eur J Radiol Open. 7:100237. doi: 10.1016/j.ejro.2020.100237

10. Treglia G (2019) Diagnostic performance of 18F-FDG PET/CT in infectious and inflammatory diseases according to published meta-analyses. Contrast Media Mol Imaging 2019:3018349. https://doi.org/10.1155/2019/3018349

11. Qin C, Liu F, Yen TC, Lan X (2020) 18F-FDG PET/CT findings of COVID-19: a series of four highly suspected cases. Eur J Nucl Med Mol Imaging 47(5):1281-1286. https://doi. org/10.1007/s00259-020-04734-w

12. Setti L, Kirienko M, Dalto SC, Bonacina M, Bombardieri E. FDG-PET/CT findings highly suspicious for COVID-19 in an Italian case series of asymptomatic patients [published online ahead of print, 2020 Apr 27]. Eur J Nucl Med Mol Imaging. 2020;1-8. doi: 10.1007/s00259-020-04819-6w

13. Albano D, Bertagna F, Bertoli $M$ et al (2020) Incidental findings suggestive of COVID-19 in asymptomatic patients undergoing nuclear medicine procedures in a high-prevalence region. J Nucl Med 61(5):632-636. https://doi.org/10.2967/jnumed.120.24625 $6 \mathrm{w}$

14. Colandrea M, Gilardi L, Travaini LL, Fracassi SLV, Funicelli L, Grana CM (2020) 18F-FDG PET/CT in asymptomatic patients with COVID-19: the submerged iceberg surfaces [published online ahead of print, 2020 Jun 23]. Jpn J Radiol. 1-5. doi: 10.1007/s11604-020-010063

15. Albano D, Camoni L, Rinaldi R, Bertagna F, Giubbini R (2020) 18F-FDG PET/CT metabolic behavior of COVID-19 pneumonia: a series of 4 patients with RT-PCR confirmation. Clin Nucl Med 45(8):e378-e380. https://doi.org/10.1097/RLU.000000000000315 0
16. Jones HA, Marino PS, Shakur BH, Morrell NW (2003) In vivo assessment of lung inflammatory cell activity in patients with COPD and asthma. Eur Respir J 21(4):567-573. https://doi. org/10.1183/09031936.03.0004850

17. Treglia G. The role of 18F-FDG PET for COVID-19 infection: myth versus reality [published online ahead of print, $2020 \mathrm{Apr} 30$ ]. Clin Transl Imaging. 2020;1-2. doi: 10.1007/s40336-020-00367-z

18. Cecconi M, Forni G, Mantovani A. Ten things we learned about COVID-19 [published online ahead of print, 2020 Jun 5]. Intensive Care Med. 2020;1-4. doi: 10.1007/s00134-020-06140-0

19. Snider SA, Margison KD, Ghorbani P et al (2018) Choline transport links macrophage phospholipid metabolism and inflammation. J Biol Chem 293(29):11600-11611. https://doi.org/10.1074/ jbc.RA118.003180

20. Olivari L, Riccardi N, Rodari P, Angheben A, Artioli P, Salgarello M (2020) COVID-19 pneumonia: increased choline uptake with 18F-choline PET/CT [published online ahead of print, 2020 Jun 4]. Eur J Nucl Med Mol Imaging. 1-2. doi: 10.1007/ s00259-020-04870-3

21. Savelli G, Bonacina M, Rizzo A, Zaniboni A. Activated macrophages are the main inflammatory cell in COVID-19 interstitial pneumonia infiltrates. Is it possible to show their metabolic activity and thus the grade of inflammatory burden with 18 F-Fluorocholine PET/CT? [published online ahead of print, 2020 May 27]. Med Hypotheses. 144:109885. doi: 10.1016/j.mehy.2020.109885

22. Maurea S, Mainolfi CG, Bombace C, et al. (2020) FDG-PET/ CT imaging during the Covid-19 emergency: a southern Italian perspective [published online ahead of print, 2020 Jun 23]. Eur J Nucl Med Mol Imaging. 1-7. doi: 10.1007/s00259-020-04931-7

23. Paez D, Gnanasegaran G, Fanti S, et al. (2020) COVID-19 pandemic: guidance for nuclear medicine departments [published online ahead of print, 2020 Apr 15]. Eur J Nucl Med Mol Imaging. 1-5. doi: 10.1007/s00259-020-04825-8

24. Tulchinsky M, Osmany S (2020) The American college of nuclear medicine guidance on operating procedures for a nuclear medicine facility during COVID-19 pandemic [published online ahead of print, 2020 May 1]. Clin Nucl Med. doi: 10.1097/ RLU.0000000000003146

Publisher's Note Springer Nature remains neutral with regard to jurisdictional claims in published maps and institutional affiliations. 